\title{
DIVISIBILITY OF GENERALIZED EXPONENTIAL AND LOGARITHMIC COEFFICIENTS
}

\author{
DONALD M. DAVIS \\ (Communicated by Frederick R. Cohen)
}

\begin{abstract}
We prove that for isomorphic formal group laws, the maximal $p$ power in the denominators of certain collections of coefficients of powers of their exponential series are equal. These numbers are significant in algebraic topology.
\end{abstract}

\section{MAIN THEOREM AND APPLICATION}

Let $R$ be a torsionfree commutative ring with 1. A formal group law (FGL) over $R$ is a formal power series

$$
F(x, y)=x+y+\sum_{i, j \geq 1} c_{i, j} x^{i} y^{i}, \quad c_{i, j} \in R,
$$

satisfying $F(F(x, y), z)=F(x, F(y, z))$. (See [Haz].) Associated to $F$ are its log series $L(x)$ and exp series $E(x)$, which are inverse to one another under composition, and satisfy

$$
F(x, y)=E(L(x)+L(y)) .
$$

The letters $L$ and $E$ will always denote $\log$ and exp series, using subscripts to specify the associated FGL, when necessary. The series $L$ and $E$ will usually have coefficients in $R \otimes \mathbf{Q}$. An important example, called the multiplicative FGL, has $R=\mathbf{Z}, F_{0}(x, y)=x+y+x y, L_{0}(x)=\log (1+x)=\sum(-1)^{i+1} x^{i} / i$, and $E_{0}(x)=e^{x}-1=\sum x^{i} / i$ !. FGL's $F_{1}$ and $F_{2}$ over $R$ are said to be isomorphic over $R$ if there is an invertible series $\phi(x) \in R \llbracket x \rrbracket$ such that

$$
\phi\left(F_{2}(x, y)\right)=F_{1}(\phi(x), \phi(y)),
$$

or, equivalently, if $E_{1}\left(L_{2}(x)\right)$ has coefficients in $R$.

Let $\operatorname{coef}\left(x^{i}, g(x)\right)$ denote the coefficient of $x^{i}$ in the series $g(x)$, and for $r \in R \otimes \mathbf{Q}$, and $p$ a prime integer, let $\nu_{p}(\operatorname{den}(r))$ denote the smallest nonnegative integer $a$ such that $p^{a} r \in R$. When $R=\mathbf{Z}$ or $\mathbf{Z}_{(p)}$, this is just the

Received by the editors August 7, 1989.

1980 Mathematics Subject Classification (1985 Revision). Primary 55N22; Secondary 10A40.

Key words and phrases. Formal group laws, James numbers, divisibility.

This work was partially supported by a National Science Foundation Research Grant DMS8900272. 
exponent of $p$ in the denominator of the rational number $r$. Here $\mathbf{Z}_{(p)}$, the integers localized at $p$, is the set of rational numbers with denominator prime to $p$. If $F$ is a FGL over $R$, we define, for $0 \leq k \leq n$,

$$
D_{p}^{F}(n, k)=\max \left\{\nu_{p}\left(\operatorname{den}\left(\operatorname{coef}\left(x^{n}, E(x)^{i}\right)\right)\right): n-k \leq i \leq n\right\},
$$

where $E$ is the exp series of $F$.

Our main theorem is

\section{Theorem.}

(i) For any FGL $F$ over $R$ with log series $L$,

$$
D_{p}^{F}(n, k)=\max \left\{\nu_{p}\left(\operatorname{den}\left(\operatorname{coef}\left(x^{j},(x / L(x))^{n+1}\right)\right)\right): 0 \leq j \leq k\right\} .
$$

(ii) If $F_{1}$ and $F_{2}$ are isomorphic FGL's over $R$, then

$$
D_{p}^{F_{1}}(n, k)=D_{p}^{F_{2}}(n, k) \text {. }
$$

For example, let $R=\mathbf{Z}_{(p)}$, and let $F_{p}$ denote the FGL with log series

$$
L_{p}(x)=\sum_{i \geq 0} x^{p^{i}} / p^{i} .
$$

It is not obvious that $F_{p}$ has coefficients in $\mathbf{Z}_{(p)}$; however, a theorem of Hasse [Ha], states that $E_{0}\left(L_{p}(x)\right) \in \mathbf{Z}_{(p)} \llbracket x \rrbracket$, from which it follows easily that, since $F_{0}$ has coefficients in $\mathbf{Z}_{(p)}$, so does $F_{p}$, and, of course, they are isomorphic. The FGL $F_{p}$ is sometimes called the canonical $p$-typical FGL. The work of this paper was undertaken to find a simple reason why $D_{p}^{F_{0}}(n, k)$ and $D_{p}^{F_{p}}(n, k)$ should be equal.

Before proving the theorem, we indicate how it was motivated by algebraic topology. We close with some explicit calculations.

\section{OCCURRENCES IN TOPOLOGY}

The first occurrence in topology of numbers like $D_{p}(n, k)$ was in [AT], where integral analogues,

$$
D(n, k)=\operatorname{lcm}\left\{\operatorname{den}\left(\operatorname{coef}\left(x^{n}, E_{0}(x)^{i}\right)\right), n-k \leq i \leq n\right\},
$$

satisfying $\nu_{p}(D(n, k))=D_{p}^{F_{0}}(n, k)$, were studied. The integral analogue of our (3) for the multiplicative FGL was proved there, by methods which we have mimicked in our proof. They prove that $D(n, k)=1$ if and only if $n+1$ is a multiple of a certain integer which is determined explicitly by $k$. The combination of $[\mathrm{AT}]$ and $[\mathrm{AW}]$ proved that the projection map of complex Stiefel manifolds $W_{n+1, k+1} \rightarrow W_{n+1,1}$ has a section if and only if $D(n, k)=1$.

The paper that first suggested (and implicitly proved) that $D_{p}^{F_{0}}(n, k)$ and $D_{p}^{F_{p}}(n, k)$ are equal was [Be]. It considered the unstable Adams-Novikov spectral sequence (UANSS) converging to the homotopy groups of the special unitary 
group $S U(r)$, and proved that for the UANSS based on complex cobordism the group $E_{2}^{1,2 n+1}(S U(r))$ was cyclic of order $n ! / D(n, n-r)$, where $D(-,-)$ is as in (4), while for the UANSS based on the $p$-local Brown-Peterson spectrum $B P$ the $p$-group $E_{2}^{1,2 n+1}(S U(r))$ was cyclic of exponent $\nu_{p}(n !)-D_{p}^{F_{p}}(n, n-r)$. Since the $p$-localization of the spectrum for complex cobordism splits as a sum of copies of $B P$, the latter $E_{2}$-term is the $p$-localization of the former, which effectively proves the equality of $D_{p}^{F_{0}}(n, k)$ and $D_{p}^{F_{p}}(n, k)$. It was a search for a simpler explanation which led to this paper.

The numbers $D(n, k)$ also appear frequently in the literature as an approximation to the stable James numbers $U^{s}(n+1, k+1)$, defined as the index of the image of the stable homotopy group $\pi_{2 n+1}^{s}\left(W_{n+1, k+1}\right)$ in the infinite cyclic group $\pi_{2 n+1}^{s}\left(W_{n+1,1}\right)$. This number equals the index of the image of the Hurewicz homomorphism for stunted complex projective space

$$
\pi_{2 n}^{s}\left(C P_{n-k}^{\infty}\right) \rightarrow H_{2 n}\left(C P_{n-k}^{\infty}\right) \text {. }
$$

One of the early references is [Lu, 3.3]; in [CK1] and [CK2] much evidence is given to support the conjecture that if $p$ is odd then

$$
D_{p}^{F_{0}}(n, k)=\nu_{p}\left(U^{s}(n+1, k+1)\right),
$$

and a modified version when $p=2$. In [CK 1, 4.2], the equality of the RHS of our (3) for the multiplicative and canonical $p$-typical FGL's is stated. We thank Michael Crabb and Karlheinz Knapp for explaining several proofs of this fact, which were adapted to our proof. We also thank Andy Baker and Martin Bendersky for pointing out the result of Hasse mentioned initially, and Francis Clarke for suggesting that an earlier version of this paper generalized to rings $R$ as above. Finally, we note that results similar to ours for Bernoulli numbers, which are related to the coefficients in $x / E(x)$, are proved in [Mi], [Di], [Ba], and $[\mathrm{Cl}]$.

\section{PROOF OF THEOREM AND A COROLLARY}

We begin by proving part (i) of the theorem, mimicking [AT, 5.1]. Write

$$
(x / L(x))^{n+1}=\sum_{i \geq 0} a_{i} x^{i},
$$

let $x=E(y)$, and, for any $j \geq 0$, multiply by $y^{n+1} E(y)^{-j-1} E^{\prime}(y)$, where $E^{\prime}(y)$ denotes the derivative, and $E(y)^{-j}$ is interpreted as $y^{-j}(E(y) / y)^{-j}$, obtaining

$$
\begin{aligned}
E(y)^{n-j} E^{\prime}(y) & =y^{n+1} \sum_{i \geq 0} a_{i} E(y)^{i-j-1} E^{\prime}(y) \\
& =y^{n+1}\left(a_{j} E(y)^{-1} E^{\prime}(y)+\sum_{i \neq j} a_{i} \frac{d}{d y}\left(\frac{E(y)^{i-j}}{i-j}\right)\right) .
\end{aligned}
$$


Since derivatives of Laurent series have no $y^{-1}$-terms, we obtain

$$
\operatorname{coef}\left(y^{n}, E(y)^{n-j} E^{\prime}(y)\right)=a_{j}=\operatorname{coef}\left(x^{j},(x / L(x))^{n+1}\right) .
$$

This could also be deduced from the Lagrange Inversion Formula [Co].

The following well-known lemma is obtained by applying $\partial / \partial y$ to (1), and then replacing $y$ by 0 , and $x$ by $E(y)$.

Lemma. If $E$ is the exp series of a FGL over $R$, then $E^{\prime}(y)=1+\sum_{i>0} \alpha_{i} E(y)^{i}$, with $\alpha_{i} \in R$.

Thus we have

$$
\begin{aligned}
\max & \left\{\nu_{p}\left(\operatorname{den}\left(\operatorname{coef}\left(x^{j},(x / L(x))^{n+1}\right)\right)\right), 0 \leq j \leq k\right\} \\
& =\max \left\{\nu_{p}\left(\operatorname{den}\left(\operatorname{coef}\left(y^{n}, E(y)^{i} E^{\prime}(y)\right)\right)\right), n-k \leq i \leq n\right\} \\
& =\max \left\{\nu_{p}\left(\operatorname{den}\left(\operatorname{coef}\left(y^{n}, E(y)^{i}\right)\right)\right), n-k \leq i \leq n\right\},
\end{aligned}
$$

establishing (i), where the first "=" follows from (5), and the second since the lemma shows that $\bmod \left(y^{n+1}\right)$ the matrix relating $\left(E^{n} E^{\prime}, \cdots, E^{n-k} E^{\prime}\right)$ to $\left(E^{n}, \cdots, E^{n-k}\right)$ is triangular with 1's on the diagonal and coefficients in $R$.

Part (ii) of the theorem is now proved by showing that the RHS of (3) for $L=L_{1}$ equals that for $L=L_{2}$. The number there could be expressed as

$$
\min \left\{a: p^{a}(x / L(x))^{n+1} \in R \llbracket x \rrbracket /\left(x^{k+1}\right)\right\} .
$$

In an expression such as this we automatically truncate $\bmod x^{k+1}$. Let $\phi(x)=$ $E_{1}\left(L_{2}(x)\right) \in R \llbracket x \rrbracket$. Then $f(x) \mapsto f(\phi(x))$ defines an isomorphism of pairs of rings

$$
\left(R \otimes \mathbf{Q} \llbracket x \rrbracket /\left(x^{k+1}\right), R \llbracket x \rrbracket /\left(x^{k+1}\right)\right) \rightarrow\left(R \otimes \mathbf{Q} \llbracket x \rrbracket /\left(x^{k+1}\right), R \llbracket x \rrbracket /\left(x^{k+1}\right)\right)
$$

for any $k$, and it sends $p^{a}\left(x / L_{1}(x)\right)^{n+1}$ to $p^{a}\left(\phi(x) / L_{2}(x)\right)^{n+1}$ for any $a$ and $n \geq 0$. In particular, one of these is in $R \llbracket x \rrbracket /\left(x^{k+1}\right)$ if and only if the other is. Because $(\phi(x) / x)^{n+1}$ is a unit in $R \llbracket x \rrbracket, p^{a}\left(\phi(x) / L_{2}(x)\right)^{n+1}$ is in $R \llbracket x \rrbracket /\left(x^{k+1}\right)$ if and only if $p^{a}\left(x / L_{2}(x)\right)^{n+1}$ is.

The theorem implies that $\left\{E_{p}^{n-k}, \cdots, E_{p}^{n}\right\}$ and $\left\{E_{0}^{n-k}, \cdots, E_{0}^{n}\right\}$ have the same maximal $p$-power of denominators of coefficients of $x^{n}$, but it is not always true that

$$
\nu_{p}\left(\operatorname{den}\left(\operatorname{coef}\left(x^{n}, E_{p}^{i}\right)\right)\right)=\nu_{p}\left(\operatorname{den}\left(\operatorname{coef}\left(x^{n}, E_{0}^{i}\right)\right)\right),
$$

even when both are nonzero. The first disparity when $p=3$ occurs when $i=4$ and $n=20$.

It is also not always true that if $F$ is strictly isomorphic over $\mathbf{Z}_{(p)}$ to $F_{0}$, then the nonzero coefficients of $E$ have the same powers of $p$ in their denominators as those of $E_{0}$. For example, let $p=3$ and compare coefficients of $x^{4}$ in $E=E_{0}+E_{0}^{2}$ and $E_{0}$. However, this will be true for a family of FGL's containing $F_{p}$. 
Corollary. If $F$ is a FGL isomorphic to $F_{0}$, and the coefficients of $x^{i}$ in $E$ (and hence also $L$ ) are 0 unless $i \equiv 1 \bmod p-1$, and if $i<p$ and $n \equiv i \bmod p-1$, then

$$
\nu_{p}\left(\operatorname{den}\left(\operatorname{coef}\left(x^{n}, E^{i}\right)\right)\right)=\nu_{p}(n !) .
$$

Thus $E+\cdots+E^{p-1}$ has coefficients which agree $p$-adically with those of $E_{0}$.

Proof. We will show that

$$
D_{p}^{F_{0}}(n, n)=\nu_{p}(n !)
$$

and if $p \leq i \leq n$, then

$$
\nu_{p}\left(\operatorname{den}\left(\operatorname{coef}\left(x^{n}, E_{0}(x)^{i}\right)\right)\right)<\nu_{p}(n !) .
$$

Hence, $D_{p}^{F_{0}}(n, n-p)<D_{p}^{F_{0}}(n, n)$, and so, by our theorem,

$$
D_{p}^{F}(n, n-p)<D_{p}^{F}(n, n)
$$

for any $F$ isomorphic over $\mathbf{Z}_{(p)}$ to $F_{0}$. If $E$ is as in the corollary, then the only value of $i<p$ for which $\operatorname{coef}\left(x^{n}, E(x)^{i}\right)$ could be nonzero, and hence account for the difference guaranteed by (8), is the unique value of $i \equiv n \bmod p-1$.

To prove (6) and (7), it is useful to define numbers $a(n, k)$ by

$$
E_{0}(x)^{k}=\left(e^{x}-1\right)^{k}=\sum a(n, k) \frac{x^{n}}{n !} .
$$

Considering the derivative shows that these satisfy $a(n+1, k)=k(a(n, k)+a(n, k-1)), \quad a(n, 1)=1, a(1, k)=0, k>1$, which shows inductively that they are integers and multiples of $p$ if $k \geq p$. (6) and (7) now follow easily, since

$$
\operatorname{den}\left(\operatorname{coef}\left(x^{n}, E_{0}(x)^{i}\right)\right)=\frac{n !}{a(n, i)}
$$

\section{Formulas FOR $D_{3}^{F_{0}}(n, k)$}

We have made extensive computer calculations of the numbers $D_{3}^{F_{0}}(n, k)$ in hopes of finding a way of determining them by inspection from $n$ and $k$. Actually, it is

$$
e(2 m-1,2 s)=\nu_{3}((2 m-1) !)-D_{3}^{F_{0}}(2 m-1,2 m-1-2 s)
$$

which we have tabulated, for, following [Be], the UANSS for 3-primary homotopy groups of the symplectic group $S p(s)$ will have

$$
E_{2}^{1,2 n+1}(S p(s))= \begin{cases}\mathbf{Z} / 3^{e(n, 2 s)} & n \text { odd } \\ 0 & n \text { even } .\end{cases}
$$

We hope to prove in a subsequent paper that the $p$-primary $v_{1}$-periodic homotopy groups $v_{1}^{-1} \pi_{2 n}(S p(s))$ (see [DM] for the definition) are given by the obvious analogue of (9). 
An example of the type of formula we desire is given by

Proposition. $e(2 m-1,20)=\max _{5 \leq k \leq 10}\left\{\min \left\{2 k+2, \nu_{3}\left(m-k-b_{k}\right)+8\right\}\right\}$, where $b_{7}=2 \cdot 3^{7}, b_{10}=3^{13}$, and other $b_{k}=0$.

Periodicity results such as those of [CK2, 3.12, 3.15] reduce verification of this proposition to a finite number of cases. We have obtained similar formulas for $e(2 m-1,2 s)$ for $s \leq 12$. The result for $s=10$ in our proposition is simpler than most, but they all suggest

Conjecture. There are integers $s_{0}, a_{k}, h_{k}$, and $b_{k}$ such that

$$
e(2 m-1,2 s)=\max _{s_{0} \leq k \leq s}\left\{\min \left\{a_{k}, \nu_{3}\left(m-k-h_{k}\right)+b_{k}\right\}\right\} .
$$

The value of $s_{0}$ will be roughly $s / 2, a_{k}$ roughly $2 k$, and $b_{k}$ roughly $s$.

\section{REFERENCES}

[AT] M. F. Atiyah and J. A. Todd, On complex Stiefel manifolds, Proc. Cambridge Phil. Soc. 56 (1960), 342-353.

[AW] J. F. Adams and G. Walker, On complex Stiefel manifolds, Proc. Cambridge Phil. Soc. 61 (1965), 81-103.

[Ba] A. Baker, Combinatorial and arithmetic identities based on formal group laws, SpringerVerlag Lecture Notes in Math., vol. 1298 (1987), 17-34.

[Be] M. Bendersky, Some calculations in the unstable Adams-Novikov spectral sequence, Publ. RIMS 16 (1980), 739-766.

[Cl] F. Clarke, The universal Von Staudt theorems, Trans. Amer. Math. Soc. 316 (1989).

[Co] L. Comtet, Advanced combinatorics, Reidel, 1974.

[CK1] M. Crabb and K. Knapp, James numbers, Math. Ann. 282 (1988), 395-422.

[CK2] - The Hurewicz map on stunted complex projective spaces, Amer. J. Math. 110 (1988), 783-809.

[DM] D. M. Davis and M. Mahowald, $v_{1}$-periodic homotopy groups of $S p(2), S p(3)$, and $S^{2 n}$, (to appear in Proc. Kinosaki Conference in Algebraic Topology).

[Di] I. Dibag, An analogue of the Von Staudt-Clausen theorem, J. Algebra 87 (1984), 332-341.

[Ha] H. Hasse, Die Gruppe der $p^{n}$-primären Zahlen für ein Primteiler von $p$, J. Reine Angew. Math. 176 (1936), 174-183.

[Haz] M. Hazewinkel, Formal groups and applications, Academic Press, 1978.

[Lu] A. T. Lundell, Generalized e-invariants and the numbers of James, Quart. J. Math. Oxford Ser. 25 (1974), 427-440.

[Mi] H. R. Miller, Universal Bernoulli numbers and the $S^{1}$-transfer, Current Trends in Algebraic Topology, CMS-AMS, 1982, pp. 437-449.

Department of Mathematics, Lehigh University, Bethlehem, Pennsylvania 18015 